\title{
Application of ATOVS Radiance with ARW WRF/GSI Data Assimilation System in the Prediction of Hurricane Katrina
}

\author{
J. Xu ${ }^{*}, 1$, S. Rugg ${ }^{2}$, M. Horner ${ }^{2}$ and L. Byerle ${ }^{2}$ \\ ${ }^{I}$ University Corporation for Atmospheric Research (UCAR), USA \\ ${ }^{2}$ Air Force Weather Agency (AFWA), USA
}

\begin{abstract}
In this study, we evaluated the impact of directly assimilating radiance on Hurricane Katrina forecasts over the Gulf of Mexico in the southeastern United States in August 2005. The ATOVS (i.e., The Advanced Television and Infrared Observation Satellite (TIROS)-N Operational Vertical Sounder) radiance data, the Gridpoint Statistical Interpolation (GSI) three-dimensional variational analysis (3DVAR) system, and the Advanced Research WRF (ARW WRF) model were employed. The results in a series of experiments show that after radiance data assimilation, the intensity and structure of initial fields including atmospheric flow, temperature and moisture have been modified somehow, especially with instruments using microwave bands such as AMSU-A/B. An anomalous southward pressure gradient has been added behind the hurricane center, which made the easterly flow go through the initial vortex center, accelerating westward movement of the hurricane. All data assimilation experiments obtain a similar forecast for the hurricane track before $36 \mathrm{~h}$ of model integration. After $36 \mathrm{~h}$, the hurricane tracks in AMSU-A/B experiments are closer to the best track, but the tracks in HIRS 3 and control experiments have a bigger error. However, we note that the improvement is limited, all assimilation experiments did not properly depict the deepening of the hurricane center around 1800 UTC 28 August.
\end{abstract}

\section{INTRODUCTION}

The assimilation of satellite radiance observations into a numerical weather prediction (NWP) system is an important path to improve weather forecasts by providing initial conditions that are more representative of the true state of the atmosphere. Preliminary impact studies of satellite data using satellite retrieved winds, and humidity were focused on the global system. The results shows a positive impact of satellite data on numerical weather prediction, especially in the Southern Hemisphere [1-6] The satellite data are a useful data source not only in global models but also in regional models. Bouttier and Kelly [7] demonstrated that the impact of rawinsonde data on the forecast was extremely large over the regional areas but the aircraft and satellite data seemed to have a little effect. The satellite data assimilation shows a strong regional feature. The impact of satellite data in regional models in North American regions, including the North American Continent surrounded by the ocean, is not very clearly. The purpose of this paper is to evaluate the impact of satellite observation data assimilation on the simulation used in a regional model.

There are two basic approaches to assimilate satellite information into a data assimilation system (DAS). The first approach is to assimilate retrieved data from radiances measured by satellite instruments. The satellite retrievals such as humidity and, wind field usually were provided by the satellite data producer independent of the data assimilation system. The second approach is to assimilate radiance measurements directly into a DAS. Direct radiance assimilation is theoretically superior to retrieval assimilation because

*Address correspondence to this author at the Joint Center for Satellite Data, Assimilation (JCSDA), 5200 Auth Road, WWB, Camp Springs, MD 20746, USA; E-mail: Jianjun.xu@noaa.gov the observational error statistics are more justified in direct radiance assimilation than in retrieval assimilation [8-11]. This approach differs from the traditional practice of transforming the observations into analysis variables and requires an observation operator built into the DAS to transform model variables into radiances. The linkage between forecast model state variables, such as temperature and humidity, and observed radiances is expressed mathematically by a forward radiative transfer model (RTM), which calculates radiance from model state vertical profiles.

Compared to global model, regional model with data assimilation is being slowly developed to some extent, due to the complication by local and diabatic effects, complex nonlinear balance relationships, and the presence of lateral boundaries [12]. The complex relationships between the different atmospheric fields and various scales of motion require a dynamical approach to data analysis and assimilation [13]. Regional models often contain information on structures associated with the local terrain, so to extract information from them satisfactorily, a model with high resolution is necessary.

Solely due to this kind of complication and request from operational forecasting for regional scales, the satellite data assimilation for initialization of a regional model has received the greatest attention. Therefore, in this study, we expect to discuss the role of satellite observations for regional modeling through a hurricane case study. Several studies investigated the effect of satellite-retrieval products (such as rain rate) on hurricane initialization and prediction [14-17]. The effect of the direct use of satellite radiance, instead of their retrieved products, needs to be assessed.

Therefore, the main emphasis for this study is to investigate how the direct assimilation of radiance into a full phys- 
ics mesoscale model affects the simulation of hurricane evolution. In this paper, the NCEP's Gridpoint Statistical Interpolation (GSI) analysis system including the prototype Community Radiative Transfer Model (pCRTM) was linked to the ARW WRF mesoscale system and the ATOVS observation used. In section 2, the ARW WRF regional model, the GSI data assimilation system and ATOVS datasets are described. Section 3 gives a case view of Katrina Hurricane. The experiments' design is presented in section 4. The impact of ATOVS data assimilation on the brightness temperature, initial condition and 72 hour weather forecast are evaluated in sections 5, 6 and 7, respectively. Finally, section 8 provides a summary and discussion.

\section{THE MODEL ANALYSIS SYSTEM AND SATEL- LITE DATA}

\subsection{ARW WRF Regional Model}

The weather model used in this study is the WRF model $[18,19]$, which is a nonhydrostatic, fully compressible, primitive equation model. Lead institutions involved in the effort include the National Center for Atmospheric Research (NCAR), Air Force Weather Agency (AFWA), National Centers for Environmental Prediction (NCEP), National Oceanic and Atmospheric Administration (NOAA), and other government agencies and universities. WRF is built around a software architectural framework in which different dynamical cores and model physics packages are presented under the same code. With the WRF model, it is possible to mix and match the dynamical cores and physics packages of different models to optimize performance since each model has strengths and weaknesses in different areas.

\subsection{GSI 3DVAR Data Assimilation System for ARW WRF Regional Model}

The Gridpoint Statistical Interpolation (GSI) analysis system is being developed based on NCEP current threedimensional variational analysis (3DVAR) system known as Spectral Statistical Interpolation (SSI) [20, 21]. The SSI has the advantage that the statistics of background error, both structure and amplitude, can be easily obtained and applied in the analysis procedure. It is simpler to apply a diagonal background error covariance in spectral space than to convolve the corresponding smoothing kernel with the innovations in physical space. However, with only a diagonal covariance in spectral space, the structure function is limited to being geographically homogeneous and isotropic about its center $[20,22]$. One has little control over the spatial variation of the error statistics when a simplified diagonal background error covariance in spectral space is used. With some computational cost associated with extra transforms in and out of the physical space in each iteration of the optimization solver, spatially inhomogeneous, for example, latitudedependent, variances can be applied, but it is not as easy to construct inhomogeneous and/or anisotropic shapes for the covariance profiles in spectral space. The GSI will overcome this kind of shortcoming.

The current GSI regional analysis system accepts NCEP's Nonhydrostatic Mesoscale Model (NMM) WRF and NCAR's ARW WRF mass core, and the input data could be either binary or netcdf format datasets. Interface is specialized separately for the WRF NMM core and the WRF mass core. For the ARW WRF mass core, the input/output are made on a B-grid, the mass variables $(T, Q)$ do not need to be interpolated, but wind variables do. $U$ is interpolated in $\mathrm{X}$ axis to mass points and $\mathrm{V}$ is interpolated in $\mathrm{y}$-axis to mass points. However, this is minimized by interpolating only the analysis increment back to the original grid and adding to the input guess.

All interpolations are linear in each direction, the projection information is not required. The code automatically determines the local scale information needed for transforming from global coordinates to local coordinates, properly rotating winds to the model frame, and $\mathrm{dx}$, dy needed for local derivatives. All of this is determined from two dimension fields available on both NMM and ARW mass core files giving the earth latitude and longitude of each model grid point, and grid dx, dy for every point.

Eventually, GSI can be connected to other models in a systematic way. Part of this has already been accomplished by eliminating the need to specify map projections for the horizontal domain definition.

Analysis system produces an analysis through the minimization of an objective function given by

$\mathrm{J}=1 / 2\left(\mathrm{x}^{\mathrm{T}} \mathrm{B}^{-1} \mathrm{x}\right)+(\mathrm{Hx}-\mathrm{y})^{\mathrm{T}} \mathrm{R}^{-1}(\mathrm{Hx}-\mathrm{y})$

where $\mathrm{x}$ is a vector of analysis increment, $\mathrm{B}$ is the background error covariance matrix, $\mathrm{y}$ is innovation vector, $\mathrm{y}=$ $\mathrm{y}_{\text {obs }}-\mathrm{Hx}_{\text {guess, }} \mathrm{R}$ is the observational and representativeness error covariance matrix, and $\mathrm{H}$ is the transformation operator from the analysis variable to the form of the observations.

Other than isotropic and homogeneous B used in SSI, GSI allows for non-homogeneous and anisotropic B formulation [23], distinguishes between land and sea and the tropics and midlatitudes, and is easy to use in both global and regional applications. The minimization algorithm is composed of two outer iterations to account for weak nonlinearities in the cost function that are complex to include in the minimization. In the first external iteration the first guess is a 6-h forecast, while in the second one it is the solution from the previous outer iteration. Currently background error cannot change in outer iteration (due to preconditioning in inner iteration). The background error variances, which vary by wavenumber and vertical mode, are fixed in time and estimated from scaled differences between 45 24-h and 48-h forecasts valid at the same time [20]. For the ARW WRF regional system, the background error statistics uses the same vertical grid structure as the first guess. The background error covariance matrix is extracted through the interpolation of NCEP's Global Forecast System (GFS) counterpart.

The observation error covariance matrix $(\mathrm{R})$ should not only contain information on the observational error but also errors in representativeness [13]. Thus, this matrix includes the error in the radiative transfer modeling. The specification of this matrix is difficult. It is clear that the errors are probably correlated spatially because of the errors in the radiative transfer, instrument errors and errors arising from imperfect cloud clearing, emissivity correction, and other components. However, these correlations are probably quite different from the spatial correlations found in the temperature and moisture retrievals and are currently not well known. For this 
reason, the GSI system has chosen these errors to be spatially uncorrelated. In addition, because the interchannel error correlations are not known, they have been set equal to zero.

For the radiance data, the transformation operator is more complex. The temperature, moisture and pressure on the Gaussian grid are bilinearly interpolated in the horizontal to the observation location to create a temperature and moisture profile.

\subsection{Radiative Transfer Model - pCRTM}

The US Joint Center for Satellite Data Assimilation (JCSDA) has developed its beta version of the Community Radiative Transfer Model (CRTM). The current version used in GSI 3DVAR data assimilation is referred to as prototype CRTM (pCRTM), which is generated from a fast radiative transfer model known as Optical Path Transmittance (OPTRAN). The OPTRAN model [24] computes atmospheric transmittance by predicting, by means of regression, the absorption coefficient for each absorbing species on the absorber path for that species. This methodology distinguishes OPTRAN from other fast transmittance algorithms such as those used in the Radiative Transfer Television InfraRed Observation Satellite (TIROS) Operational Vertical Sounder (RTTOVS), which predicts optical depth at fixed pressure levels. This approach was chosen because the increments of absorber amount can be selected such that the variation in transmittance is less than that of pressure increments. The advantages of performing regression on absorber levels rather than on pressure levels are threefold: (1) pressure is available for use as a predictor, (2) zenith angle is implicitly included in the absorber profile and no longer needs to be treated explicitly and (3) an arbitrary input pressure profile is permitted, so interpolation to specific pressure levels is not required. The detailed algorithm of OPTRAN can be found in the publication by McMillin et al. [25].

\subsection{ATOVS Data}

The ATOVS radiance data was supplied by NESDIS. The ATOVS is composed of Advanced Microwave Sounding Unit (AMSU) and High-Resolution Infrared Sounder (HIRS/3). Two separate radiometers (AMSU-A and AMSUB) compose the AMSU platform. The AMSU-A is a crosstrack, stepped-line scanning total power radiometer. The instrument has an instantaneous field-of-view of $3.3^{\circ}$ at the half-power points providing a nominal spatial resolution at nadir of $48 \mathrm{~km}$. The AMSU-B is a cross-track, continuous line scanning, the total power radiometer with an instantaneous field-of-view of $1.1^{\circ}$ (at the half-power points). Spatial resolution at nadir is nominally $16 \mathrm{~km}$. The antenna provides a cross-track scan, scanning $\pm 48.95^{\circ}$ from nadir with a total of 90 earth fields-of-view per scan line.

\section{CASE VIEW}

Hurricane Katrina formed from a tropical wave and became a depression about 175 miles southeast of Nassua in the Bahamas on August 23, 2005. It became a tropical storm in the following day. It then moved northwestward through the Bahamas before turning westward toward southern Florida and gradually strengthened. The storm became a category 1 hurricane and made landfall on the Miami Dade/Broward county line during the evening of August 25th. After cross- ing southern Florida and entering the Gulf of Mexico, the storm began to strengthen, reaching category 5 status on August 28th about 250 miles south-southeast of the mouth of the Mississippi River. Its winds reached their peak intensity of $175 \mathrm{mph}$ and pressure fell to $902 \mathrm{mb}$. The storm turned northwest and then north, making landfall in Plaquemines Parish, Louisiana just south of Buras with $140 \mathrm{mph}$ winds (category 4) at 6:10 am on August 29th. Continuing northward, Katrina made a second landfall near the Louisiana/Mississippi border at 10:00 am with maximum winds near $125 \mathrm{mph}$ (category 3). Then Katrina weakened as it moved to the north-northeast, but was still a hurricane 100 miles inland near Laurel, Mississippi. The storm continued to weaken and became a tropical depression near Clarksville, Tennessee on August $30^{\text {th }}$.

\section{EXPERIMENTS DESIGN}

In the ARW WRF regional model, the physics of the model include the WRF Single Moment (WSM) 3-class simple ice microphysics scheme [26], Yosei University planetary boundary layer (PBL) scheme [27], 5-layer thermal diffusion land surface scheme, Rapid Radiative Transfer Model (RRTM) longwave radiation [28], and the Dudhia shortwave radiation scheme [29]. The 12-km WRF model forecast with a mesh size domain of $456 \times 350$ (Fig. 1) was used. The vertical 50-layer $\sigma$ values are $0.997,0.990,0.982,0.973,0.962,0.951$, $0.938,0.923,0.906,0.887,0.865,0.841,0.816,0.787,0.756$, $0.722,0.686,0.648,0.608,0.569,0.531,0.495,0.461,0.429$, $0.398,0.369,0.341,0.316,0.291,0.268,0.245,0.224,0.204$, $0.186,0.169,0.152,0.136,0.121,0.108,0.095,0.082,0.071$, $0.060,0.050,0.041,0.032,0.024,0.016,0.009$, and 0.003 .

The experiment design consists of five simulations that are summarized in Fig. (2). For the purpose of eliminating the effect of radiance in the first guess field from NCEP analysis data, we generated a spin-up run for 6 hours from 1800 UTC 26 August to 0000 UTC 27 August 2005. Then the control (CTRL) experiment was made with initial conditions at 0000 UTC 27 August 2005 and run for 72 hours. The lateral boundary conditions came from the operational GFS forecast at 6-hour intervals. For the four data assimilations experiments (RATOVS, RAMSUA, RAMSUB, RHIRS3), we first used the GSI data assimilation system with different datasets, e.g., ATOVS (AMSU-A+AMSU-B+HIRS/3), AMSU-A, AMSU-B and HIRS/3, respectively, to modify the initial condition at 0000 UTC 27 August 2005, then ran the ARW WRF forecast model the same way as with the controlled experiment. In order to conveniently compare to the no assimilation (CTRL) experiment and concisely describe the common characteristics of the assimilation experiments, the four radiance assimilation experiments are referred to uniformly as radiance assimilation (RASSIMI). There are a total of 7 available sensors, including $\mathrm{AMSU}-\mathrm{A} / \mathrm{B}$ in NOAA-15, AMSU-A/B and HIRS/3 in NOAA-16, and the AMSU-B and HIRS/3 in NOAA-17 used in this study.

\section{IMPACT ON THE BRIGHTNESS TEMPERATURE}

In this section, innovation vectors (i.e., observed minus background radiance) of brightness temperature is used to estimate the capability of the radiative transfer model. For the GSI system in the current study, an observation preprocessor has been developed which includes bias correction and quality control (QC). 


\section{Model Domain}

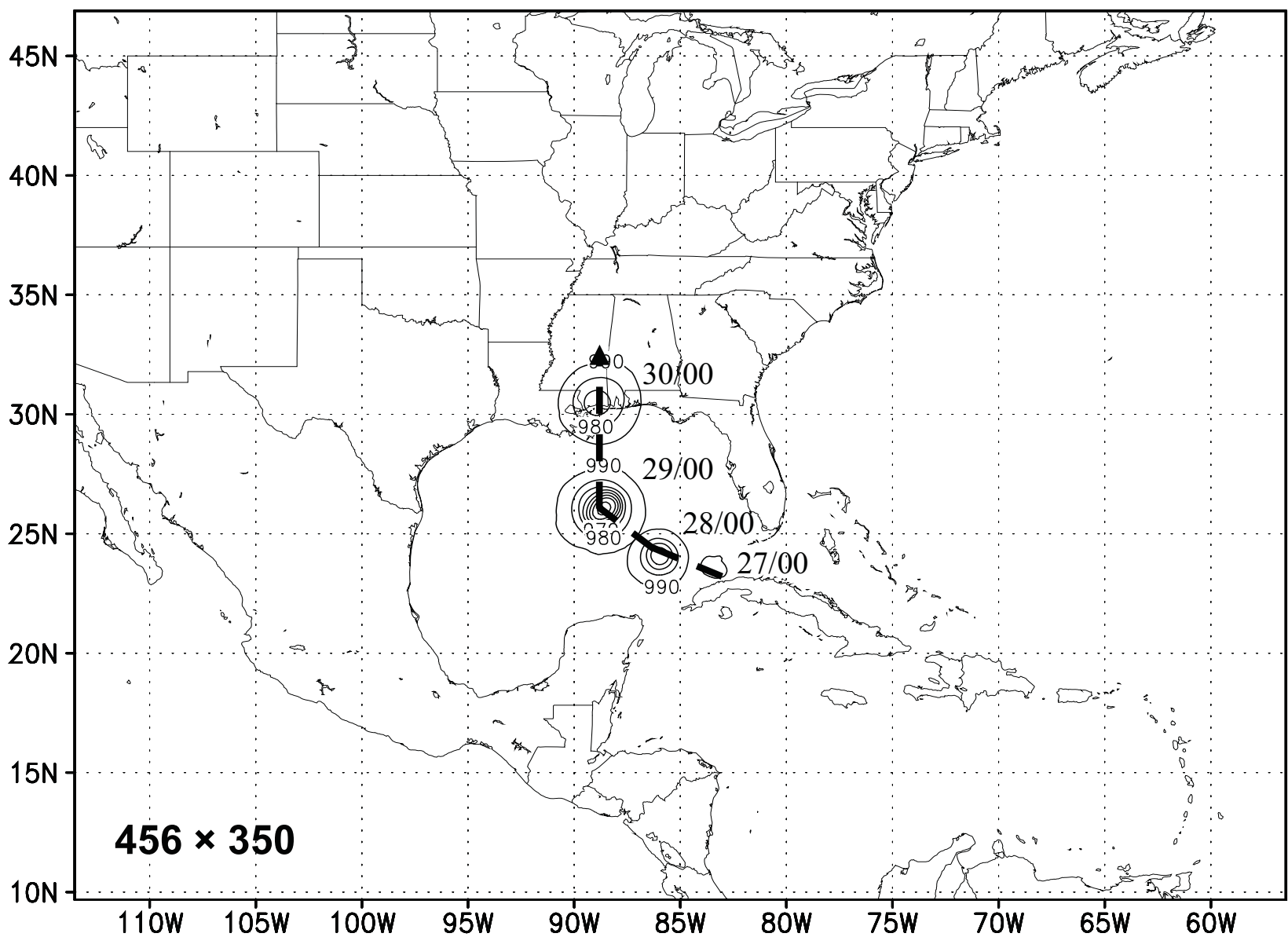

Fig. (1). The model domain used in forecast experiments, and the track of Hurricane Katrina during 0000 UTC 27 through 0000 UTC 30 August, 2005.

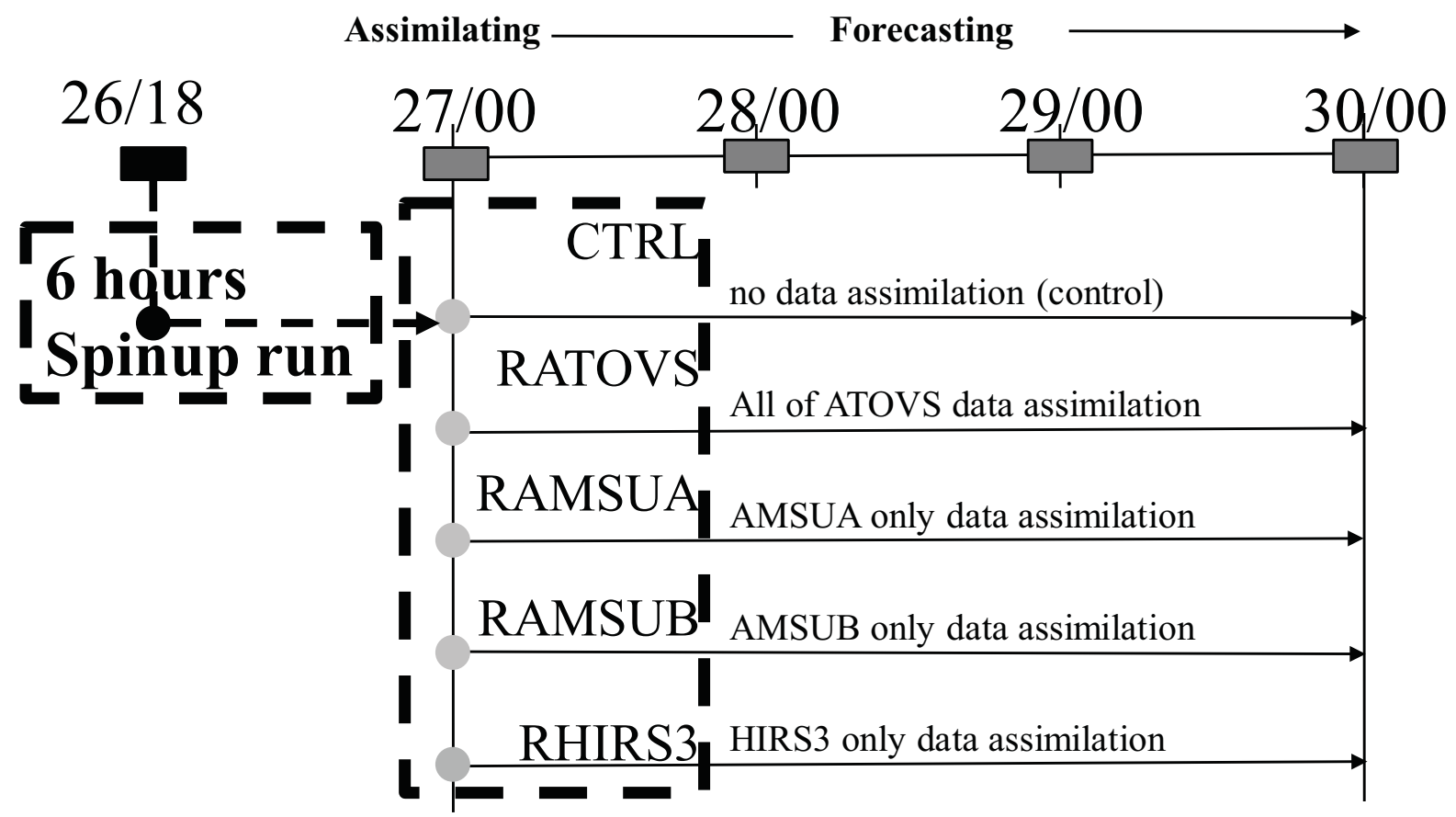

Fig. (2). Experiments design including CTRL, RATOVS, RAMSUA, RAMSUB and RHIRS3 and indicate the radiance used in assimilation and subsequent forecast. The 6 hours spinup run was conducted from 0018 UTC 26 through 0000 UTC 27 August 2005. 


\subsection{Bias Correction}

These data have undergone substantial preprocessing by NESDIS before becoming available for usage. The data have been statistically limb corrected (adjusted to nadir) and surface emissivity corrected in the microwave channels (AMSU-A and AMSU-B) and cloud cleared in the tropospheric channels (HIRS/3). This processing is referred to as bias correction. The source of the biases can be related to instrument calibration problems, and predictor and zenith angle bias. The biases are not constant for each channel, and dependent on satellite sensor. For example, Fig. (3) shows a comparison of innovations as a function of the channels, and the evidence is observed that the innovations for the whole regional set of observations are reduced using bias correction. With bias correction, the innovation for most channels is quite small, less than $1^{\circ}$ in terms of brightness temperature, and its magnitude is much smaller than the value in no bias correction. So, the best way to account for the bias is to remove all satellite calibration problems, remove all ground processing problems, and improve the radiative transfer. However, this is not feasible in the short term [9]. Practically, the effects of the spatially dependent biases can be estimated at the observation locations and removed from the data. The scheme for the bias correction is a simple linear equation for each satellite and each channel based partially on Eyre [30]. As predictors of the bias, the GSI system has chosen scaled values of a constant term, the solar zenith angle, the approximate local zenith angle (the mean of the angle for the bin), and the square of the approximate local zenith angle. These predictors are multiplied by a set of coefficients to produce the bias correction.

\subsection{Quality Control}

Derber and $\mathrm{Wu}[9]$ pointed out that a presence of a single data point containing large errors can result in substantial degradation of the analysis and subsequent forecast. For this reason, a simple quality control has been developed and the observed brightness temperature data have been modified empirically with various parameters for different instruments. In the GSI analysis system, the check will include two steps. First, a location check (including removal of observations outside the domain) and thinning procedure (ex- cluding location/time duplicates and incomplete observations) will be performed to ensure vertical consistency of upper-air profiles. Secondly, numerous quality control (QC) checks are redone based on various quality parameters after the modeled brightness temperature was obtained through the radiative transfer model. These quality parameters are used in terms of the expected observational error variance as a function of channels and have been adjusted by the position across the track of the scan, whether it is over land, sea, snow, sea ice, a transition region, elevation, the difference between the model and the real topography, and the latitude. For example, in AMSU-B channel 2, the quality parameter has been modified by the error of transmittance at higher topography (greater than 2000 meters) and the differences between the window channel observations or the simulated window channels, the brightness temperature innovation vector without QC (Fig. 4), the maximum of innovations in the three satellites (NOAA-15, NOAA-16 and NOAA-17) get to $130 \mathrm{~K}, 90 \mathrm{~K}$ and $70 \mathrm{~K}$, respectively, in some position. In contrast, with QC, the maximum innovation value is about 4 to $5 \mathrm{~K}$. Thus, it is not difficult to illustrate that quality control is very important for the use of any type of data.

Unfortunately, because of incomplete quality parameters, some good data has been rejected. In Fig. (5), the statistics show that the number used in the GSI regional data assimilation system is quite different. The AMSU-B has much more than the other two AMSU-A and HIRS/3 instruments. Firstly, we will look at the details for each channel. In NOAA-15 (Fig. 5a), the maximum number of AMSU-B for all 5 channels is over 12,000 pixels, and for AMSU-A, the number is only around 4, 000 pixels. In NOAA-16, there are only several hundred pixels in HIRS $/ 3$ and AMSU-A, while the number is a little more in AMSU-B. In NOAA-17, the number in AMSU$\mathrm{B}$ gets to about 3,000, while the other instruments are almost zero. Note that the HIRS/3 in NOAA-15 and AMSU-A in NOAA-17 are not available. Meanwhile, the root mean squared error (RMSE) and standard deviation between observation and simulated radiance varied with different instruments in terms of channel function. On the average, the error in AMSU-B is a little higher than the other two instruments (Fig. 5b).

Bias (Obs-Model) of Brightness Temperature

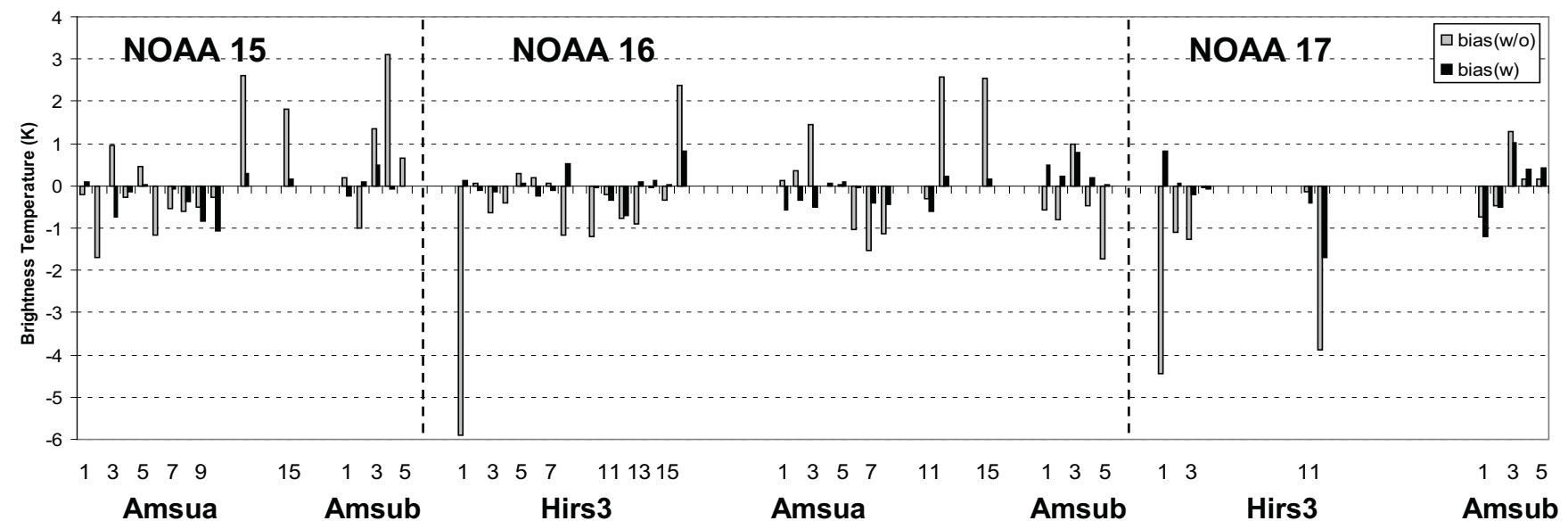

Fig. (3). Innovation (OBS minus Model) of brightness temperature changes with the channels and instruments. 

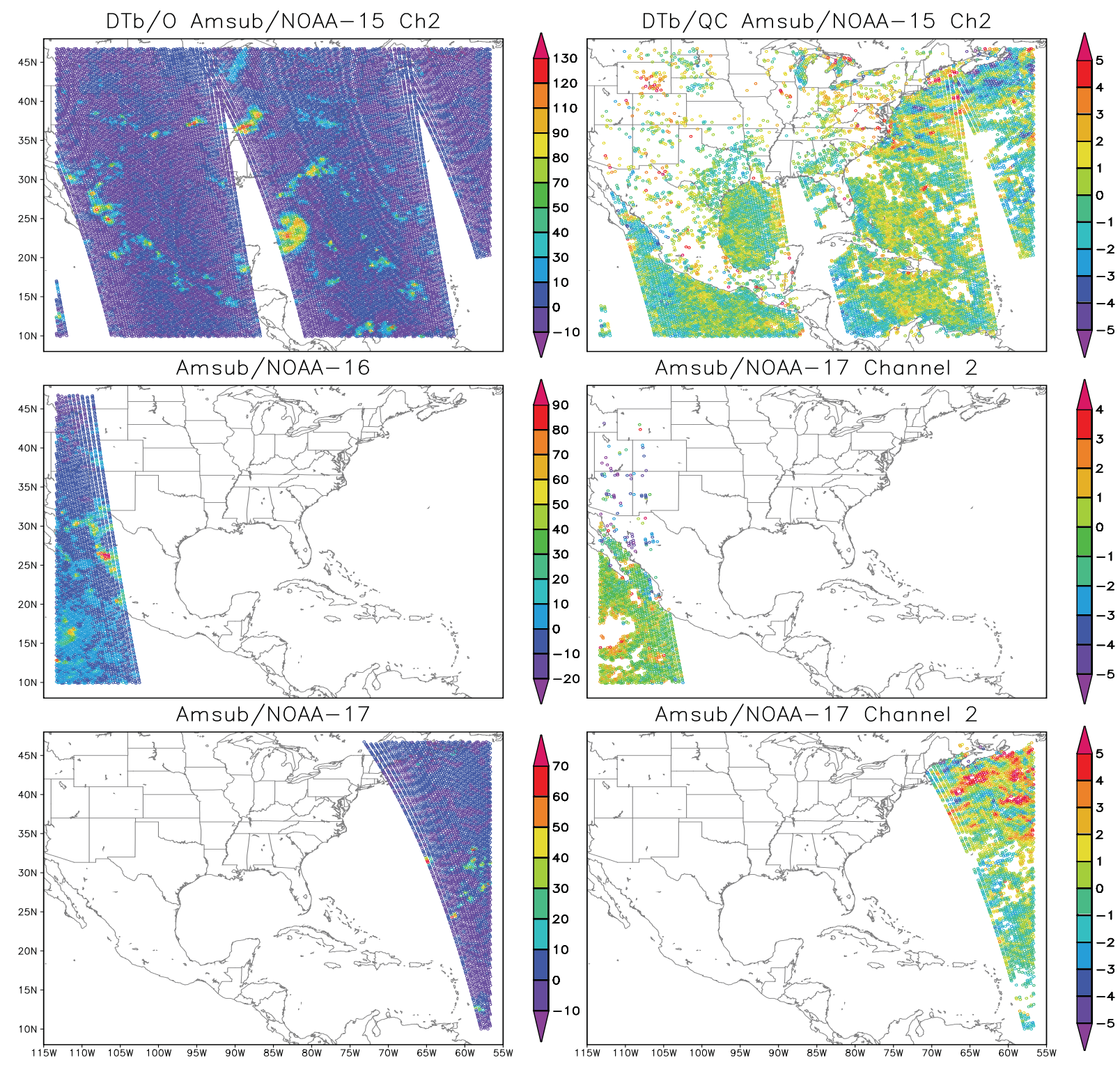

Fig. (4). Innovation of brightness temperature in Channel 2, AMSUB, Left panel with no QC and right panel after QC.

\section{IMPACT ON THE INITIALIZATION}

Before discussing the impact of ATOVS radiance data assimilation on the initial condition, we first look at the preliminary feature of initial fields in the control experiments (CTRL) without data assimilation. In order to clearly understand the detailed information about Hurricane Katrina, we focus on the domain near the hurricane vortex region and surrounding areas within a distance of around 1000 kilometers beyond the hurricane eye. At the initial time (0000 UTC 27 August 2005), there was a very strong vortex located over the southeastern Gulf of Mexico with a maximum wind speed of $45 \mathrm{~m} / \mathrm{s}$ at $850 \mathrm{hPa}$ (Fig. 6a). The sea level pressure in the vortex center falls down to $987 \mathrm{hPa}$. Over the western portion of the system, not far from the vortex, there is a closed geopotential high height center of $592 \mathrm{gpm}$ at $500 \mathrm{hPa}$ (Fig. 6b). Consistent with the lower pressure cyclone center, a warm (Fig. 6c) and wet (Fig. 6d) airmass exists around the center. The air is cold and dry over the northeastern side, and warm and wet over the northwest side. A pressure-longitude cross section through the hurricane center $\left(23.5^{\circ} \mathrm{N}\right)$ shows (Fig. 7a) the initial vertical structure of the hurricane has a substantial asymmetric feature--the zonal wind component at upper levels $(200 \mathrm{hPa})$ on the east side of the hurricane eye has a stronger westerly with a speed of $20 \mathrm{~m} / \mathrm{s}$. Under this level, the wind was dominated by weaker easterlies. It appears that a strong convergent flow does not exist near the hurricane eye. This is quite different from the cyclone over the middle latitude areas. In contrast, the meridional wind 

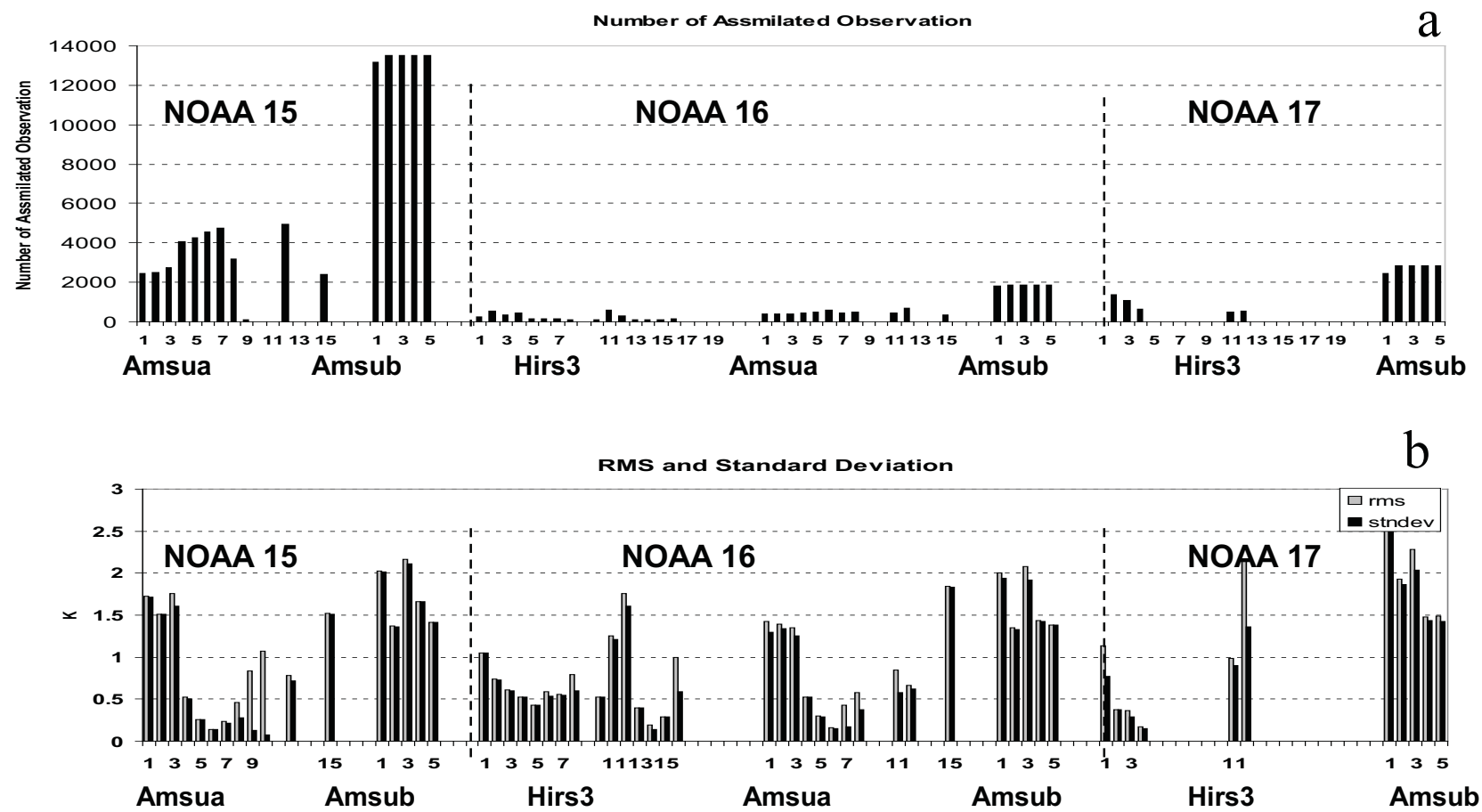

Fig. (5). The observational number (a) and rms error, standard deviation (b) of radiance depend on the channels of different instruments in the data assimilation processes.

component at lower levels appears to have a typical asymmetric structure with southerly (northerly) winds on the east (west) side of the central hurricane (Fig. 7b). The highest temperature appears inside of the hurricane eyewall, but it disappears above $500 \mathrm{hPa}$ (Fig. 7c). However, the largest specific humidity predominates the hurricane eye (Fig. 7d) under the $500 \mathrm{hPa}$ level. Therefore, it is obvious that warm temperatures do not completely coincide with high-moisture regions. This kind of temperature and moisture distribution reflects the asymmetric structure of the hurricane.

We now consider a question: what happens with radiance initialization? First of all, for the wind field, compared to the analysis with no radiance assimilation (marked as CTRL), in all radiance assimilation experiments (marked as RASSIMI), including RATOVS, RAMSUA, RAMSUB and RHIRS3, the configuration of the initial vortex is similar. The location, asymmetric ring and radius of maximum winds didn't change greatly (solid shading in Fig. 8), but the wind field increment (defined as RASSIMI minus CTRL and same as in following) is quite different. For the wind speed (shaded), the maximum increment reaches $-1.8,-1.2,1.2$, and $0.2 \mathrm{~m} / \mathrm{s}$ in RATOVS, RAMSUA, RAMSUB and RHIRS3, respectively. The maximum wind speed increment appears in the different location relative to the hurricane eye, i.e., east side in RATOVS, northeast side in RAMSUB, southeast side in RAMSUA and far east side in RHIRS3. Please note the presence of a common feature: there is a negative increment in the northwest side 1500 kilometers beyond the hurricane eye. For the flow increment, in RAMSUB, a southeasterly flow prevails over the northeastern areas of the hurricane eye; a Difference Cyclonical (marked as DC) inflow enhanced the hurricane cyclonical rotation. In RAMSUA, a positive anomaly easterly flow is going through the hurricane center, a Difference Anticyclonical flow (marked as DA) appears over northeastern areas. In RATOVS, a Difference Cyclonical (DC) is observed over the northwest of the vortex center and two branches of flow increment with southwestward and northwestward go through the hurricane core. In contrast, in RHIRS3, the flow has no any significant changes.

Consistent with the changes of wind field, the pressure fields are modulated by the radiance assimilation in the same way. The sea level pressure shows (shaded in Fig. 9) in RASSIMI that the central pressure of the hurricane (988 $\mathrm{hPa}$ ) is almost the same (Fig. 9 vs Fig. 6b). The geopotential height increment at $500 \mathrm{hPa}$ is given by a contour line in Fig. (9). Here, we find that the height increment increases with latitude in all RASSIMI experiments, which forms an extra pressure gradient with a direction from north to south. This evidence is consistent with the easterly flow increment prevailing around vortex center in the wind fields (see Fig. 8). However, the pattern of the field increment changes by incorporating radiance. The maximum increment in RAMSUA, RAMSUB and RATOVS gets up to $12 \mathrm{gpm}$ and is only $0.2 \mathrm{gpm}$ in RHIRS3. The pressure gradient behind the hurricane center in RAMSUA is a little higher than that in RAMSUB. For RATOVS (Fig. 9a), a closed Difference High (marked as DH) center appears over the west side of hurricane center and made the anomalous easterly flow separate into two branch (see Fig. 8a). The Difference Low (marked as DL) over the west side of hurricane center in RAMSUB is closer to the hurricane eye than that in RAMSUA (Fig. 6a $v s$ 9b). This pattern is beneficial when moving westward of the hurricane center. 

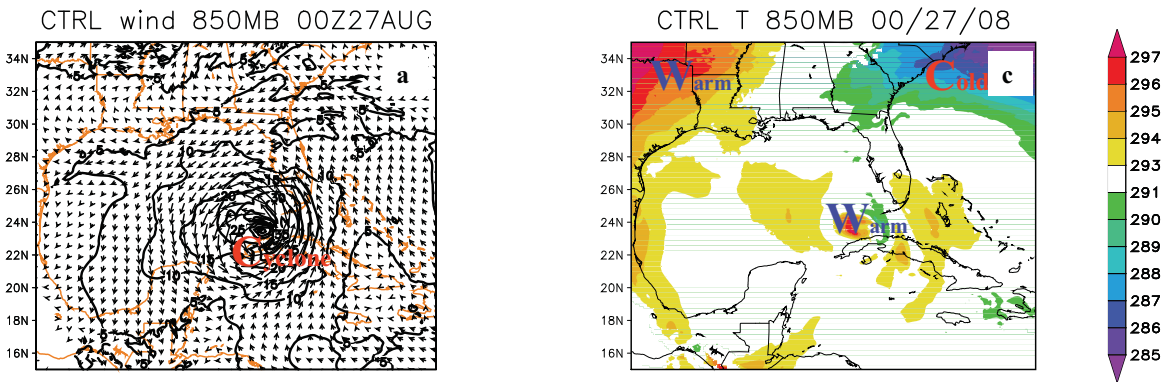

$\overrightarrow{50}$
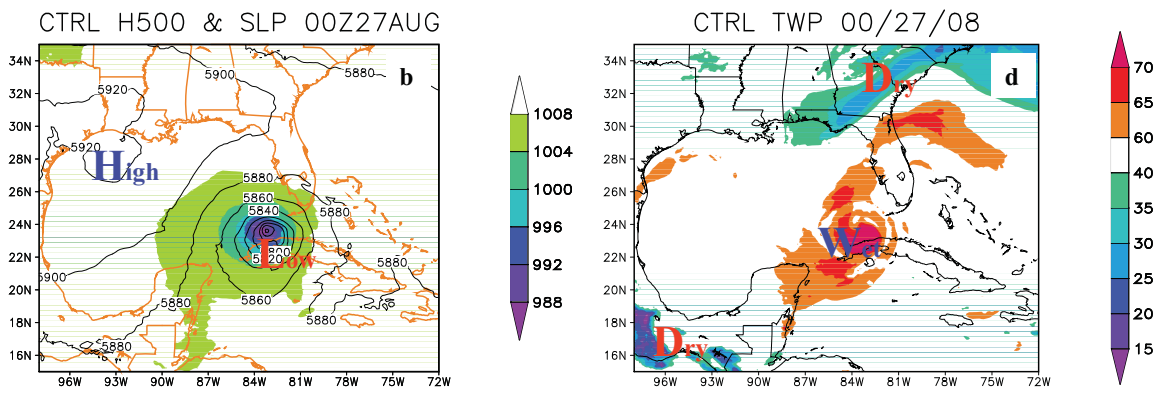

Fig. (6). Distribution of the initial wind field at $850 \mathrm{hPa}$ (a: contour is wind speed with interval of $5 \mathrm{~m} / \mathrm{s}$, vector is wind flow) variable, sea level pressure (b: shaded with maximum $1008 \mathrm{hpa}$ ), geopotential height at $500 \mathrm{hpa}$ (b: contour with interval of 20 gpm), temperature at 850 $\mathrm{hPa}$ (c), shaded with 291 through $293 \mathrm{~K}$ breaking ) and total water vapor (d, shaded with 40 through $60 \mathrm{~g} / \mathrm{Kg}$ breaking) in the CTRL experiment.
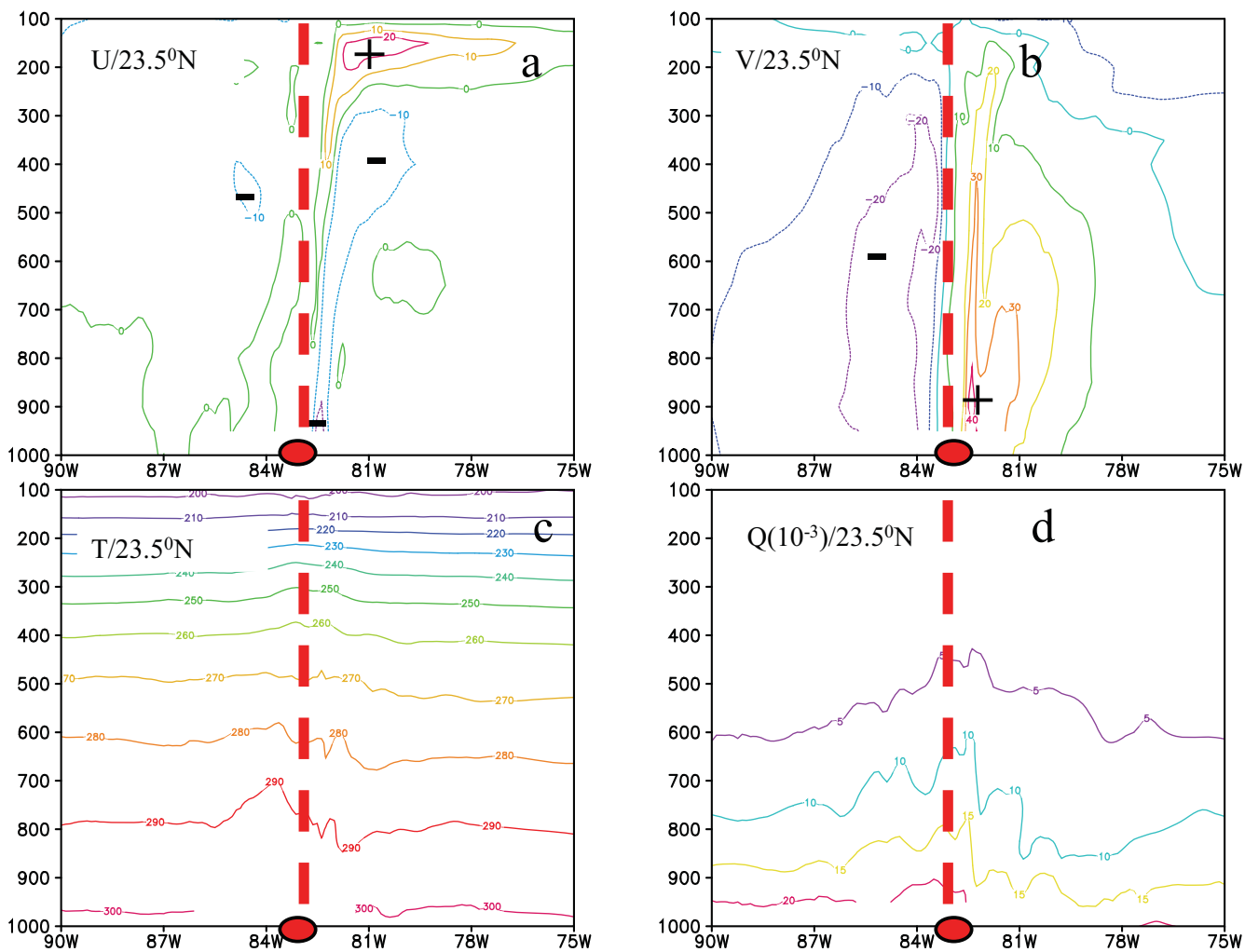

Fig. (7). Cross sections of initial zonal wind (a) and meridional wind (b) with interval of $10 \mathrm{~m} / \mathrm{s}$, temperature (c) with interval of $10 \mathrm{~K}$ and specific humidity (d) with interval of $5 \mathrm{~g} / \mathrm{Kg}$ in the CTRL experiment. 

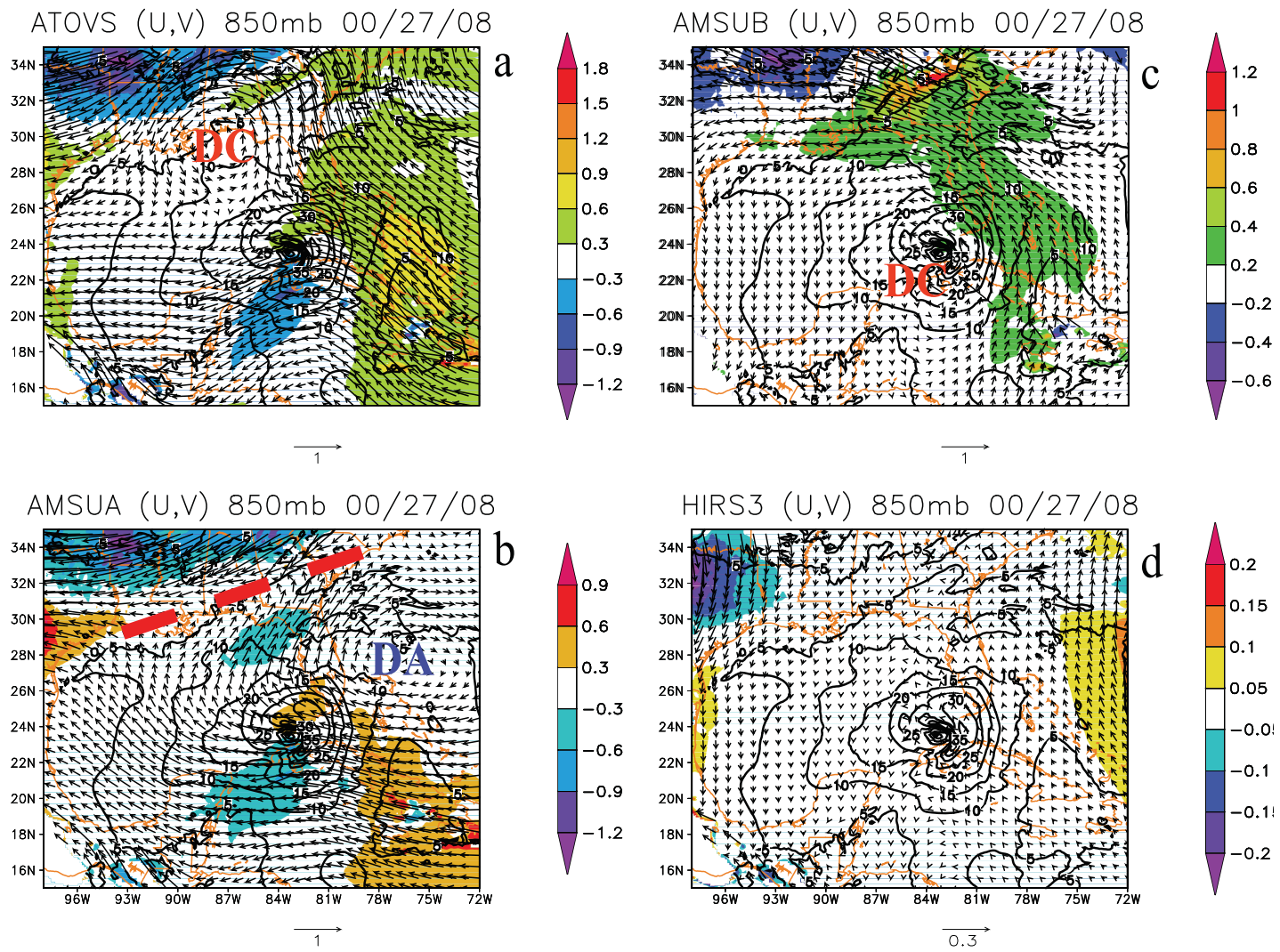

Fig. (8). Initial wind speed (contour with interval of $10 \mathrm{~m} / \mathrm{s}$ ) and wind flow increment (RASSIMI-CTRL, shaded with unit of m/s) in the assimilation experiments. DC means different cyclone, DA means different Anticyclone, heavy dished line indicates the shear convergent zone. (a) RATOVS, (b) RAMSUA, (c) RAMSUB, (d) RHIRS3.
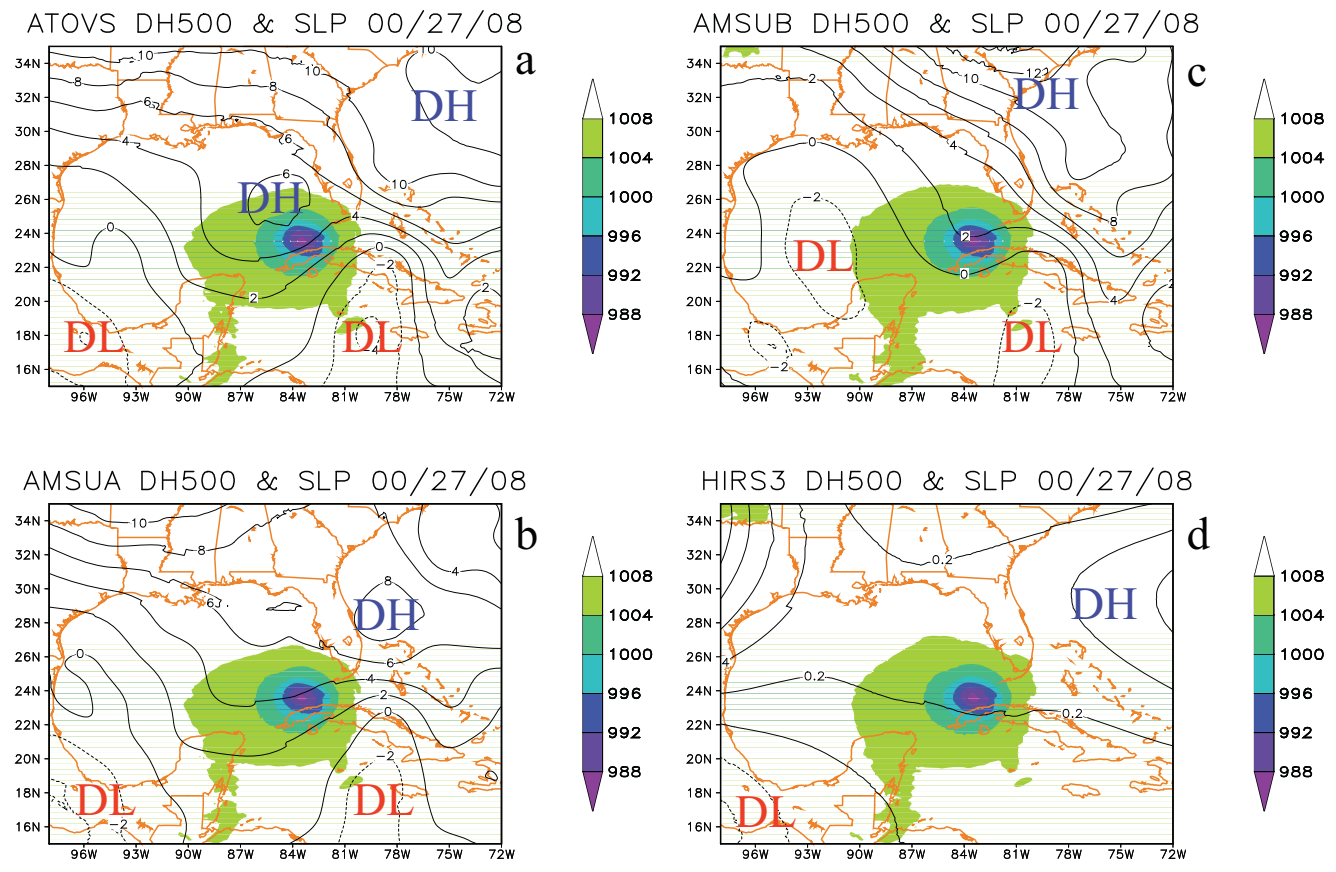

Fig. (9). Initial sea leval pressure (shade with maximum of $1008 \mathrm{hpa}$ ) and height increment (RASSIMI-CTRL) at 500 hpa (contour with interval of $2 \mathrm{gpm}$ except for HIRS3 with $0.2 \mathrm{gpm}$ ) in the assimilation experiments. DH means different high center, DL means different low. (a) RATOVS, (b) RAMSUA, (c) RAMSUB, (d) RHIRS3. 
For the temperature, the shaded coverage in Fig. (10) shows that the structure seems not to change after radiance assimilation, compared with the initial CTRL field in Fig. (6c). However, the field (contour line) increment indicates that the temperature decreases over the northern areas of the hurricane center and increases over the southern areas, which is consistent with the positive height increment to the north and negative to the south in Fig. (9). Similar to the changes in atmospheric circulation, the temperature is also changing through the use of radiance. The maximum increment gets up to $-1.8 \mathrm{~K}$ in RATOVS, RAMSUA and RAMSUB (Fig. 10a-c) and only $-0.14 \mathrm{~K}$ in RHIRS3 (Fig. 10d). The location of Difference Cold (marked as DCD) and Difference Warm (marked as DWM) centers are obviously different.

As for moisture, the total water vapor has a similar pattern (Fig. 11) in all RASSIMI experiments, but the distribution of moisture increment (the centers are marked as DDY and DWT) change substantially. To the northeast area beyond the hurricane center, there is a negative increment, but the southeast area shows a positive increment. In contrast, for RHIRS3, the increment almost equals zero (Fig. 11d).

For the vertical structure, the vertical profile of the standard deviation of temperature and specific humidity fields averaged within $400 \mathrm{~km}$ from vortex center shows that the largest changes in the temperature field are above the 300 hPa level of the model (Fig. 12a) by $0.9 \mathrm{~K}, 1.1 \mathrm{~K}, 0.6 \mathrm{~K}$ in RATOVS, RAMSUA and RAMSUB, respectively. There is no difference in RHIRS3. The largest change in the moisture field is at $850 \mathrm{hPa}$ (Fig. 12b), but the difference in RAMSUA is smaller than that in RAMSUB and RATOVS.

Based on the above analysis, we found that after the initialization with radiance, the intensity and structure of initial fields including atmospheric flow, temperature and moisture have been modified somehow, especially with instruments using microwave bands such as AMSU-A/B. An anomalous southward pressure gradient has been added behind the hurricane center, which made the easterly flow go through the initial vortex center, accelerating westward movement of the hurricane.

\section{IMPACT ON THE WEATHER FORECAST}

The effect of radiance data assimilation on the track and intensity forecast of Hurricane Katrina is first assessed by comparing observations with the predicted hurricane positions, the central sea level pressure (SLP) and the maximum low-level wind. Fig. (13) illustrates the track changes at 6-h intervals for the entire forecast period from 0000 UTC 27 to 0000 UTC 30 August 2005. The results indicate that RA-
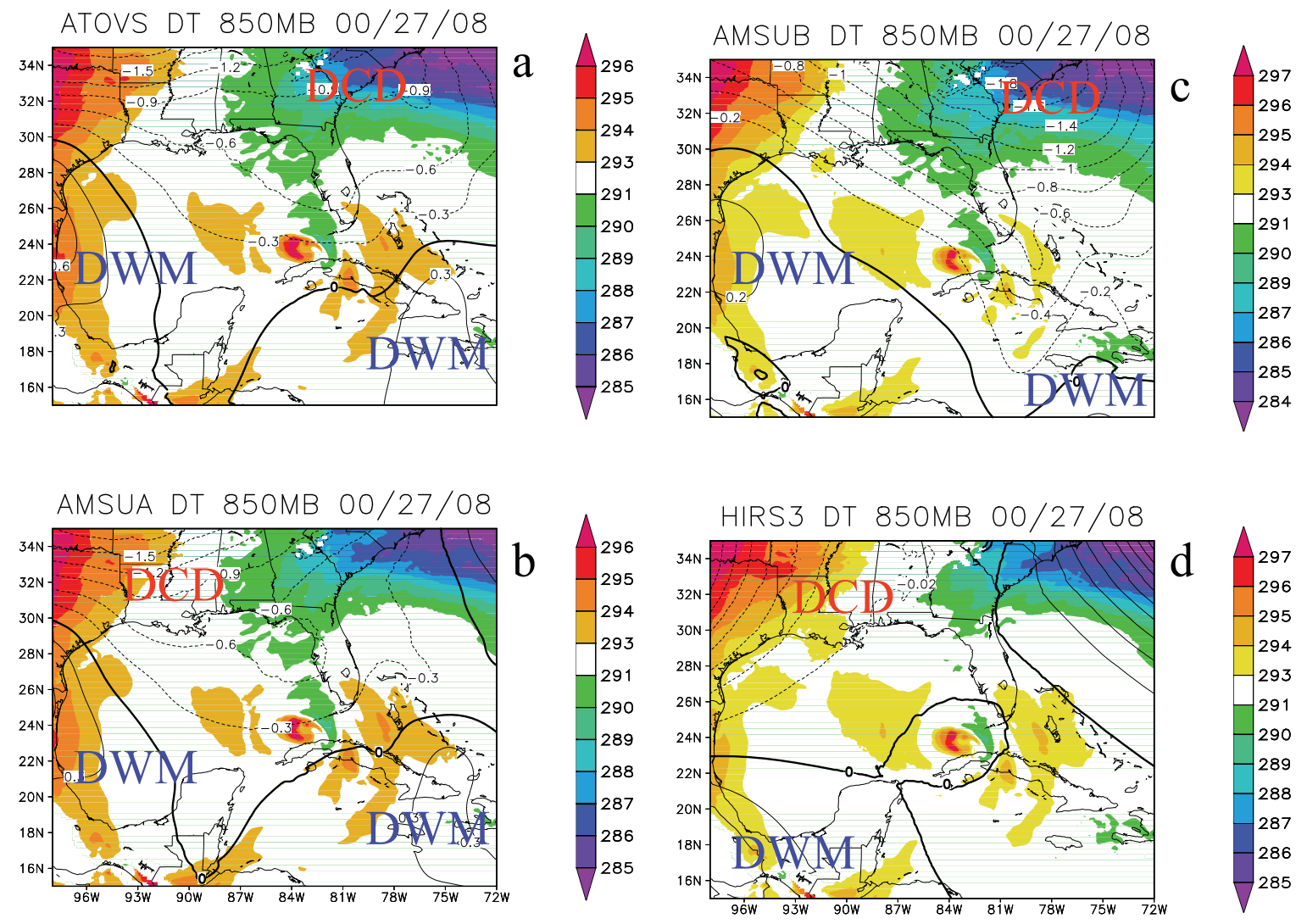

Fig. (10). Initial temperature (shaded with unit of $\mathrm{K}$ ) and temperature increment (RASSIMI-CTRL, contour with unit of K) in the assimilation experiments. DWM means different warm center, DCD means different cold center. (a) RATOVS, (b) RAMSUA, (c) RAMSUB, (d) RHIRS3. 

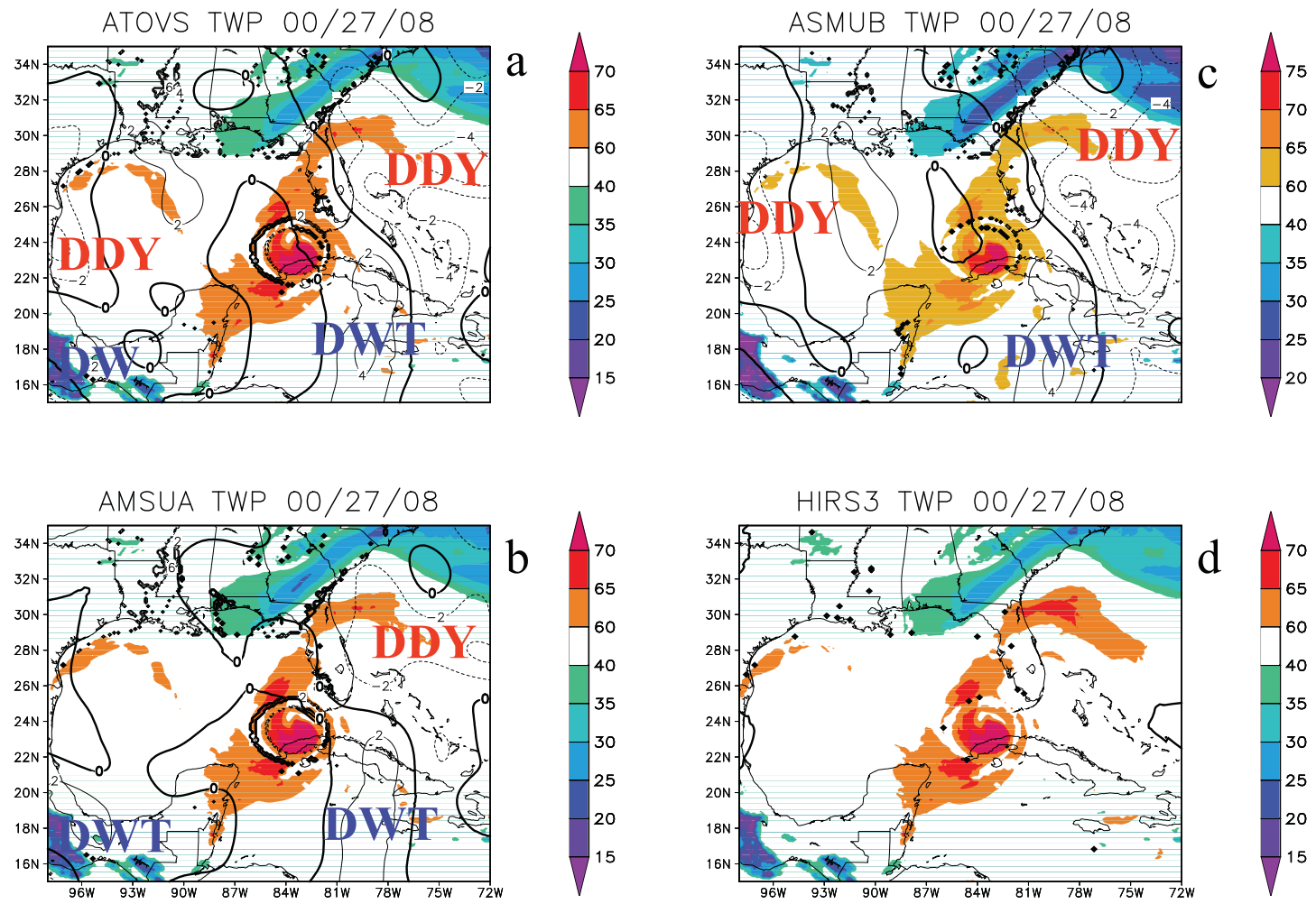

Fig. (11). Initial total vapor (shaded with 40 through $60 \mathrm{~g} / \mathrm{Kg}$ breaking) and total water vapor increment (RASSIMI-CTRL, contour with unit of $\mathrm{g} / \mathrm{Kg}$ ) in the assimilation experiments. DDY means different dry center, DWT means different wet center. (a) RATOVS, (b) RAMSUA, (c) RAMSUB, (d) RHIRS3.
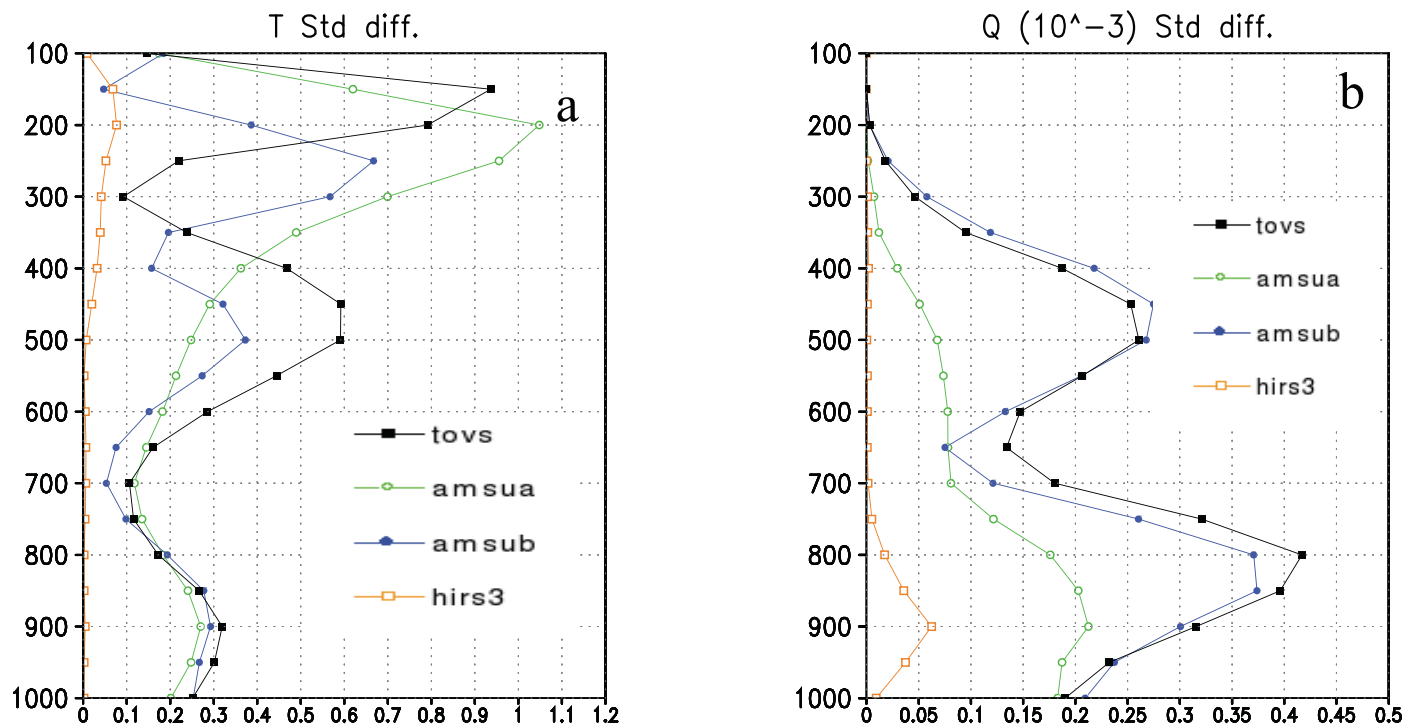

Fig. (12). The vertical profiles of temperature (a) with unit of $\mathrm{K}$ and specific humidity (b) with unit $\mathrm{g} / \mathrm{Kg}$ of standard deviation between RASSIMI and CTRL averaged over Hurricane central areas.

TOVS get a better track than the CTRL experiment does. The distance error (Fig. 14a) between the observed and simulated hurricane eye shows that the CTRL run failed to predict the position of the hurricane center after 24 hours. The forecasts with radiance assimilation produced a much improved position of prediction and subsequent northward movement that occurred between 36 and $72 \mathrm{~h}$. All experiments obtain a similar forecast for the hurricane track before $36 \mathrm{~h}$ of model integration. After $36 \mathrm{~h}$, the hurricane track in RATOVS and RAMSUB are closer to the best track, but the tracks in RHIRS3 and CTRL have a bigger error. Please note that distance error is mainly from the error in the initial field. 


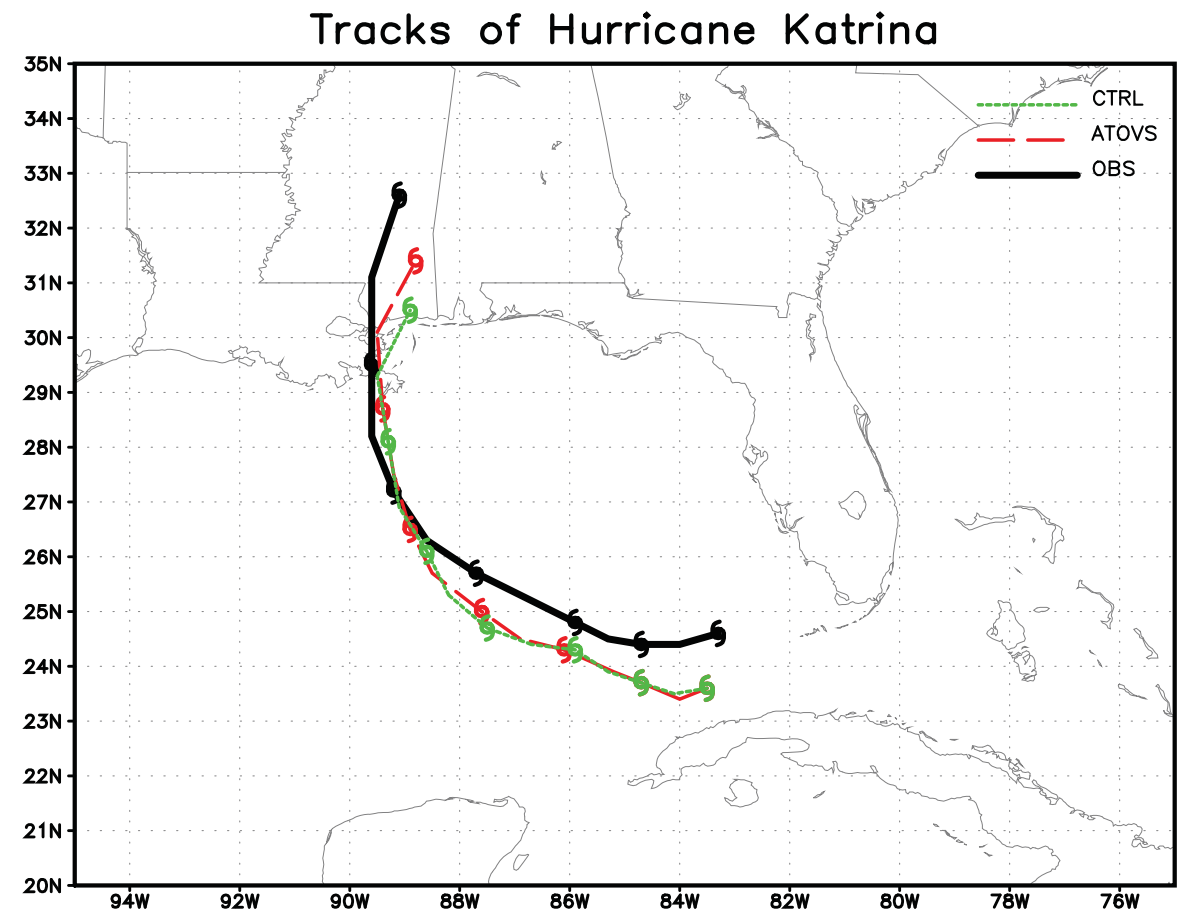

Fig. (13) Track of Hurricane Katrina Simulated initial at UTC 00 August 272005.
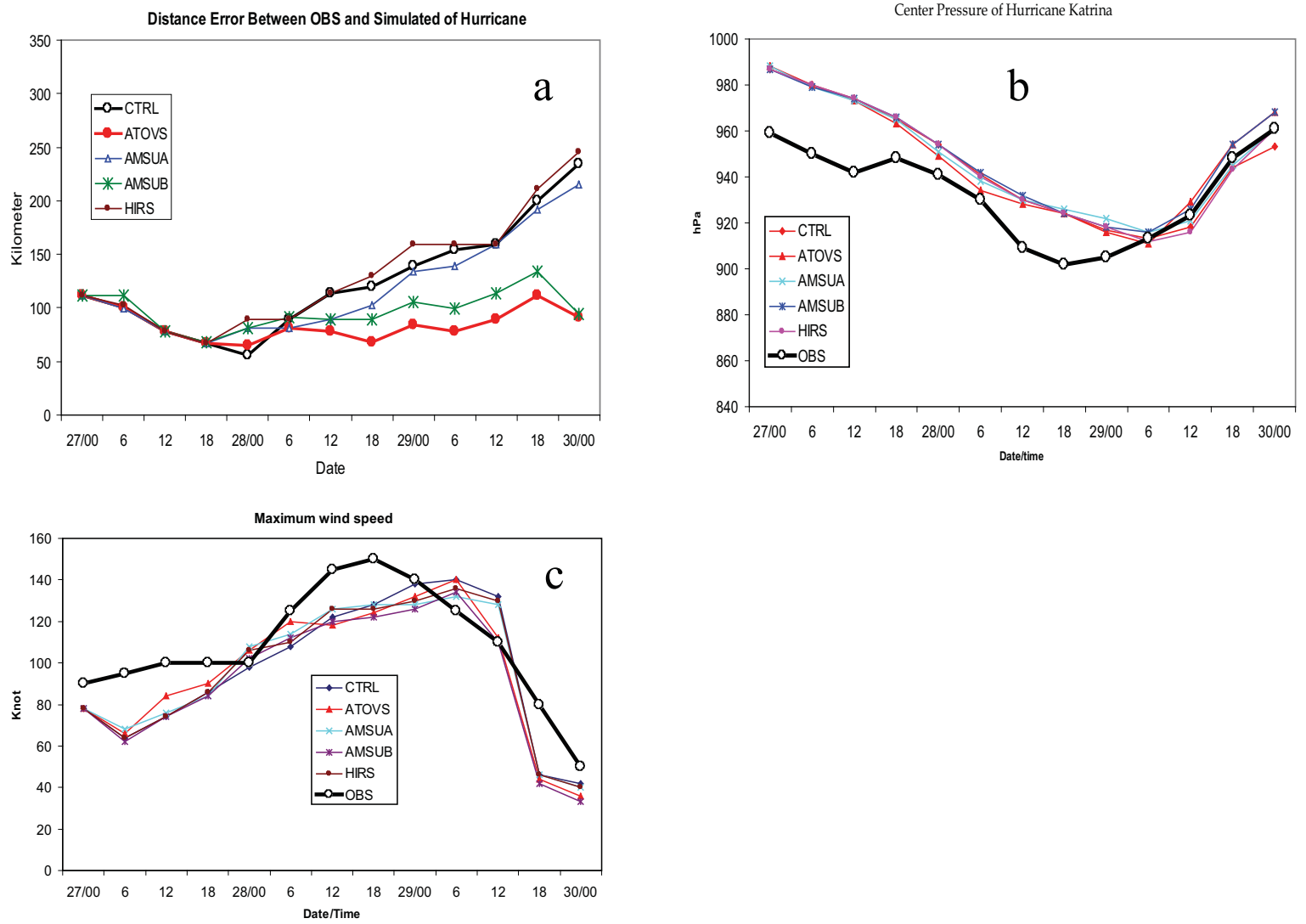

Fig. (14). Track error (a) with unit of kilometer, central pressure (b) with unit of hpa and maximum wind speed (c) with unit of knot.

The central SLP (Fig. 14b) and maximum low-level wind (Fig. 14c) were predicted at similar strength in all experiments. The maximum low-level wind and central pressure were predicted to occur by the model at 0600 UTC 29 August 2005. This time is 12 hours later than the observa- tion. The modeled central pressure is $920 \mathrm{hPa}$, which is a little higher than the observation $(902 \mathrm{hPa})$. The maximum low-level wind forecasted was 130 knots, a little lower than the observation (140 knots). 
Fig. (15) shows the upper-level wind $(200 \mathrm{hPa})$ from RATOVS (right panels) and CTRL (left panels) forecasts at 0000 UTC 28, 29, and 30 August 2005. It was observed that the wind speed of Hurricane Katrina in RATOVS was a little stronger than that in CTRL. At 0000 UTC 29 August, the 30 $\mathrm{m} \mathrm{s}^{-}{ }^{1}$ isotach to the west of the hurricane in RATOVS extends a little farther west away from the hurricane center in CTRL. We also notice that the hurricane is located in the left side of biggest wind speed core. An upper-level westerly jet resulted from the large-scale environmental response to the anticyclonic flow that fanned out of the hurricane center from all directions around the immediate environment of the hurricane. By 0000 UTC 30 August, the hurricane eye disappears. This is due to the hurricane landing.

As a comparison, Fig. (16) shows the simulated hydrometeor fields described by vertically integrated cloud water, ice, rainwater, and snow, which are the 24-h and 48-h predictions of RATOVS and CTRL. The simulated cloud distribution and the area of the vortex at 0000 UTC 16 August (Fig. 16) conform to the satellite imagery well. Both the model and the observations show the development of organized spiral cloud bands with an echo-free eye in its central core. The model also simulates well the cellular convection at the outer edge and intense, organized clouds in the
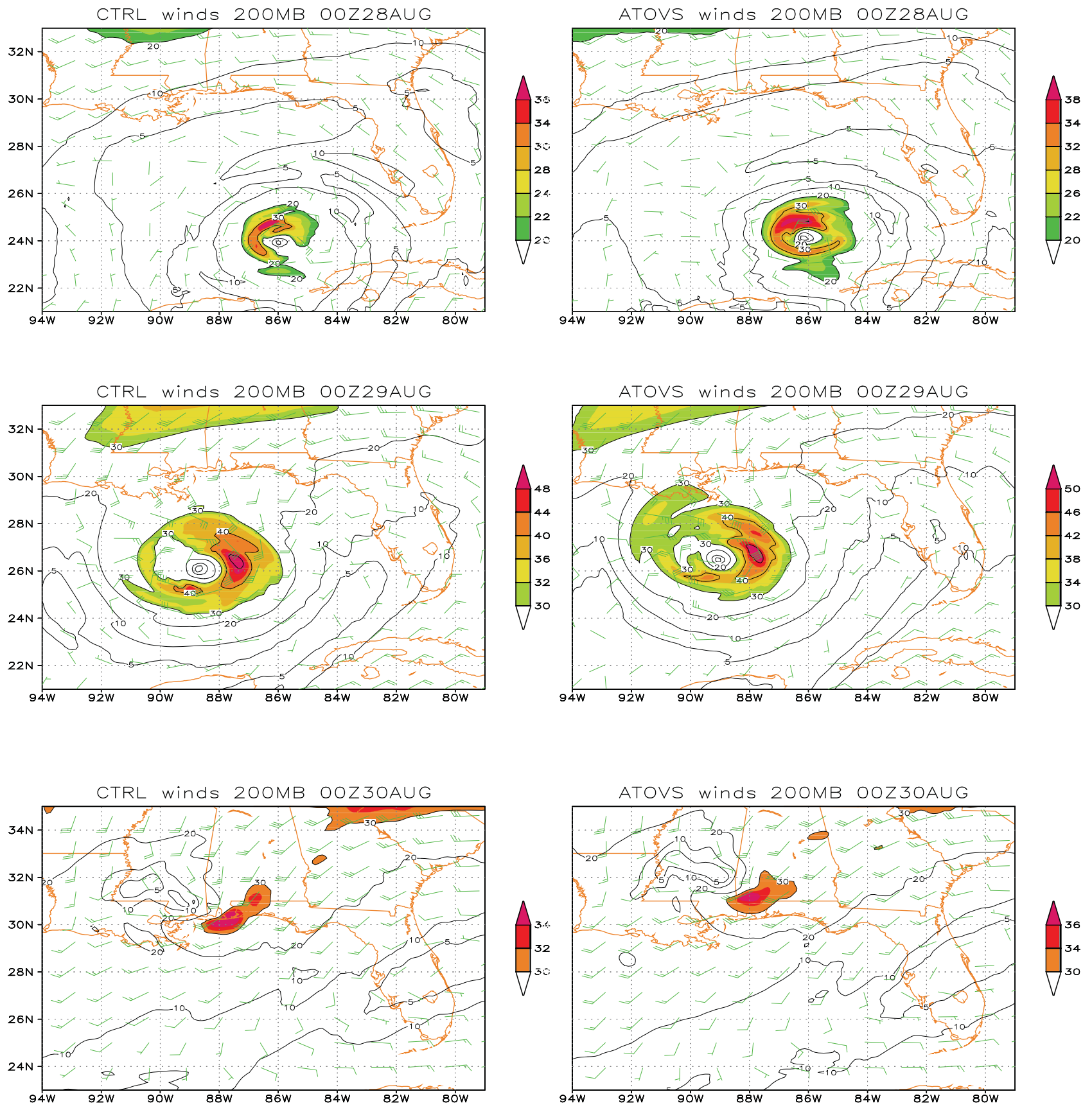

Fig. (15). The wind and wind speed (contour with unit of m/s) on $200 \mathrm{hpa}$ for 24-, 48- and 72 hour forecast, CTRL (left panel), RATOVS (right panel). The minimum of shaded is $30 \mathrm{~m} / \mathrm{s}$. 

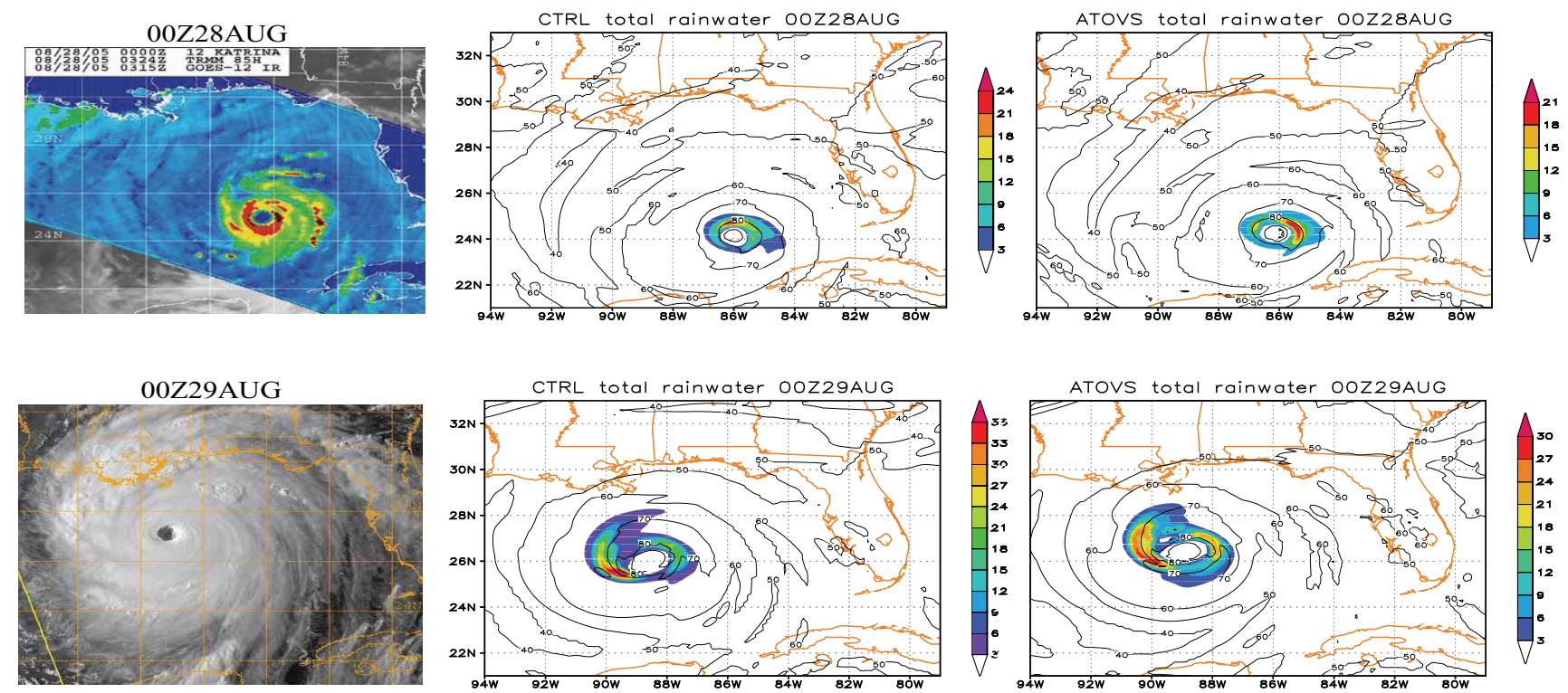

Fig. (16). Satellite imagery (left panel), integration of vapor water, cloud water and rain water in CTRL (middle panel) and RATOVS (right panel). The vapor water is displayed with contour in unit of $\mathrm{g} / \mathrm{Kg}$. The total cloud water and rain water is displayed by shaded with minimum of $3 \mathrm{~mm}$, and the black points are the location of observed hurricane eye.

eyewall. The distributions of the clouds (Fig. 16) allow us to assess the capability of model prediction in simulating the inner-core structures of Katrina. Using satellite image as a benchmark comparison, a slightly better cloud convection is found in RATOVS.

The results show that the assimilation of radiance can produce a better track of hurricane center for a forecasting period of 72 hours. It is worth noticing that the performance is different for multichannel instruments, especially for the AMSU-A/B, where the movement of Hurricane Katrina is impacted. However, we note that the improvement is limited. The RASSIMI experiment did not properly depict the deepening of the hurricane center around 1800 UTC 28 August (see Fig. 14), which is probably related to the requirement of more high model resolution [31] and it is worth to be explored in further studies.

\section{SUMMARY AND DISCUSSION}

\section{a. Summary}

We evaluated the impact of ATOVS including AMSU-A, AMSU-B and HIRS $/ 3$ radiance direct assimilation through the JCSDA pCRTM on the forecasts of Hurricane Katrina over the Gulf of Mexico in the southeastern United States in August 2005. The NESDIS ATOVS radiance data, NCEP GSI three-dimensional variational analysis (3DVAR) system and NCAR ARW WRF mesoscale system were employed in the study.

A series of experiments (RATOVS, RAMSUA, RAMSUB and RHIRS3) was conducted. The evidence indicated that a great amount of satellite data has been assimilated, especially for AMSU-B, resulting in a better forecast track. After radiance data assimilation, the intensity and structure of initial fields including atmospheric flow, temperature and moisture have been corrected somehow. For instruments with microwave bands such as AMSU-A/B, an anomalous southward pressure gradient was added on the area behind the hurricane core, which leads to large scale easterly flow going through the initial hurricane center, and accelerating the westward movement of the hurricane.

The performance of the three instruments is quite different. There are much more radiance being used in the assimilation processes in AMSU-B, which produced a more significant impact on the forecast of Hurricane Katrina than the other instruments did. It worked very well for the 72-hour forecast period. In contrast, because most of data has been kicked out under the cloud coverage, the HIRS/3 instrument did not have any significant forecast impact when compared to the no data assimilation (CTRL) forecasting.

\section{b. Discussion}

In this study, the radiance assimilation using the GSI analysis system with ARW WRF model showed a positive improvement for Hurricane Katrina forecasts over the southeastern United States. However, due to the complexity of microphysical processes in a hurricane, and the accurate selection of model resolution, as well as the lack of accurate hurricane center intensity and location error described in first guess field, and a lot of valuable observational data being tossed out through imperfect data quality control, especially areas under significant cloud coverage, as well as the background error calculation for the ARW WRF model, the limitations of radiance assimilation should be given more attention in future studies.

First of all, accurate initial condition plays a significant role in forecasting of hurricane, unfortunately, the current first guess field from NCEP global forecasts system cannot 
adequately represent the initial circulations of tropical cyclones, especially the initial position of hurricane center, the average position errors for the NCEP hurricane track forecasts were over $100 \mathrm{~km}$ for 24-h forecasts for Atlantic hurricanes. To solve this problem, there are two main approaches for hurricane initialization: (i) substitute a specified vortex circulation defined by an analytical expression for the analyzed vortex into the initial conditions [32], (ii) bogus scheme, implant a "spinup" vortex generated by the same forecast model into the initial conditions [33]. This study shows that the radiance direct assimilation modulated significantly the initial circulation (Figs. 8-11). However, it can't modify the initial position error of hurricane center (Fig. 14). So that hurricane initialization becomes a necessary step before making a prediction.

Secondly, in the current GSI data assimilation system, the cloud-cleared radiance data is permitted. Meanwhile, before the observational radiance can be used in assimilation, the data quality control is necessary, because of incomplete quality parameters, some good data has been rejected. Due to the lack of sufficient data, it is hard to represent precisely the three-dimensional inner-core structures of hurricane. In fact, hurricane change including intensity and track is closely related to the evolving three-dimensional structures of the hurricane. If more data including cloud-covered radiance data can be used, developing a new algorithm is required. Except for insufficient observations, the limitations of forecast models including low-resolution, crude physical parameterization and the inability to treat multiscale interactions are essential to be considered.

Finally, for ARW WRF regional model, the background error statistics in GSI data assimilation system use the same vertical grid structure as the first guess of NCEP global model forecasts. The background error covariance matrix is extracted through the interpolation of NCEP's GFS counterpart. The NMC-method [20] is popular method for estimating climatological background error covariance. In reality, errors in the background field will be synoptically dependent, i.e. vary from day to day depending on the current situation. It is clear that background errors should estimate errors from ARW WRF regional model for each domain. It is worth to be studied in the future work.

\section{ACKNOWLEDGEMENTS}

The first author would like to thank the Joint Center for the Satellite Data Assimilation providing the GSI data assimilation system. Special thanks are extended to Dr. Russ Treadon, Dr. Wu, Dr. Derber and Dr. Parrish for answering many questions regarding the GSI data assimilation system. The comments and suggestions offered by the three anonymous reviewers contributed to considerable improvement of the paper.

\section{REFERENCE}

[1] Tracton MS, Desmarais AJ, van Haaren RJ, McPherson RD. The impact of satellite soundings on the National Meteorological Center's analysis and forecast system-The Data Systems Test results. Mon Wea Rev 1980; 108: 543-86.

[2] Halem M, Kalnay E, Baker WE, Atlas R. An assessment of the FGGE satellite observing system during SOP-1. Bull Amer Meteor Soc 1992; 63: 407-29.

[3] Andersson E, Hollingsworth A, Kelly G, Lönnberg P, Pailleux J, Zhang Z. Global observing system experiments on operational sta- tistical retrievals of satellite sounding data. Mon Wea Rev 1991; 119: 1851-64.

[4] Mo KC, Wang XL, Kistler R, Kanamitsu M, Kalnay E. Impact of satellite data on the CDAS-reanalysis system. Mon Wea Rev 1995; 123: 124-39.

[5] Derber JC, Parrish DF, Lord SJ. The new global operational analysis system at the National Meteorological Center. Wea Forecasting 1991; 6: 538-47.

[6] Lahoz WA, O'Neill A. Impact of research satellite observations in a data assimilation system for the troposphere and stratosphere. Third WMO Workshop on the Impact of Various Observing Systems on NWP, Alpbach, Austria, 9-12 March 2004.

[7] Bouttier F, Kelly D. Observing-system experiments in the ECMWF 4D-Var data assimilation system. Quart J Roy Meteor Soc 2001; 127:1469-88.

[8] Eyre JR. A fast radiative transfer model for satellite sounding systems. ECMWF Tech Memo 1991; 176: 28 pp. [Available from European Centre for Medium-Range Weather Forecasts, Shinfield Park, Reading, Berkshire R62 9AX, United Kingdom.].

[9] Derber JC, Wu WS. The use of TOVS cloud-cleared radiances in the NCEP SSI analysis system. Mon Wea Rev 1998; 126: 2287-99.

[10] McNally AP, Derber JC, Wu W, Katz BB. The use of TOVS level$1 \mathrm{~b}$ radiances in the NCEP SSI analysis system. Quart J Roy Meteor Soc 2000; 126: 689-724

[11] Liu Q, Weng F. Detecting the warm core of a hurricane from the Special Sensor Microwave Imager Sounder. Geophys Res Lett 2006; 33: L06817, doi:10.1029/2005GL025246.

[12] Stauffer DR, Seaman NL, Binkowski FS. Use of four-dimensional data assimilation in a limited-area mesoscale model part II: effects of data assimilation within the planetary boundary layer. Mon Wea Rev 1991; 119: 734-54.

[13] Lorenc AC. Analysis methods for numerical weather prediction. Quart J Roy Meteor Soc 1986; 112: 1177-1194.

[14] Krishnamurti TN, Roy Bhowmik SK, Oosterhof D, Rohaly G. Mesoscale signatures within the tropics generated by physical initialization. Mon Wea Rev 1995; 123: 2771-90.

[15] Peng MS, Chang SW. Impacts of SSM/I retrieved rainfall rates on numerical prediction of a tropical cyclone. Mon Wea Rev 1996; 124: 1181-98.

[16] Karyampudi VM, Lai GS, Manobianco J. Impact of initial conditions, rainfall assimilation, and cumulus parameterization on simulations of Hurricane Florence (1988). Mon Wea Rev 1998; 126: 3077-101.

[17] Xiao Q, Zou X, Kuo YH. Incorporating the SSM/I-derived precipitable water and rainfall rate into a numerical model: A case study for the ERICA IOP-4 cyclone. Mon Wea Rev 2000; 128: 87-108.

[18] Michalakes J, Chen S, Dudhia J, et al. Development of a next generation regional weather research and forecast model. Developments in Teracomputing: Proceedings of the Ninth ECMWF Workshop on the Use of High Performance Computing in Meteorology, Zwieflhofer W, Kreitz N, Eds. World Scientific 2002; 269-76.

[19] Skamarock WC, Klemp JB, Dudhia J, et al. A description of the Advanced Research WRF Version 2. NCAR Tech Note NCAR/ TN-468+STR, 2005: 94 pp.

[20] Parrish DF, Derber JC. The National Meteorological Center's spectral statistical interpolation analysis system. Mon Wea Rev 1992; 120: 1747-63.

[21] Derber JC, Parrish DF, Lord SJ. The new global operational analysis system at the National Meteorological Center. Wea Forecasting 1991; 6: 538-47.

[22] Courtier P, Coauthors. The ECMWF implementation of threedimensional variational assimilation (3D-Var). I: Formulation. Quart J Roy Meteor Soc 1998; 124: 1783-807.

[23] Wu WS, Purser RJ, Parrish DF. Three-dimensional variational analysis with spatially inhomogeneous covariances. Mon Wea Rev 2002; 130: 2905-16.

[24] Kleespies TJ, van Delst P, McMillin LM, Derber JC. Atmospheric transmittance of an absorbing gas. 6. OPTRAN status report and introduction to the NESDIS/NCEP Community Radiative Transfer Model. Appl Opt 2004; 43: 3103-9.

[25] McMillin LM, Core LJ, Kleespies TJ. Atmospheric transmittance of an absorbing gas. 5: Improvements to the OPTRAN approach Appl Opt 1995; 34: 8396-99.

[26] Hong SY, Dudhia J, Chen SH. A revised approach to ice microphysical processes for the bulk parameterization of clouds and precipitation. Mon Wea Rev 2004; 132: 103-20. 
[27] Hong SY, Pan HL. Nonlocal boundary layer vertical diffusion in a medium-range forecast model. Mon Wea Rev 1996; 124: 2322-39.

[28] Mlawer EJ, Taubman SJ, Brown PD, Iacono MJ, Clough SA. Radiative transfer for inhomogeneous atmosphere: RRTM, a validated correlated-k model for the longwave. J Geophys Res 1997; 102 (D14): 16663-82.

[29] Dudhia J. Numerical study of convection observed during the winter monsoon experiment using a mesoscale two-dimensional model. J Atmos Sci 1989; 46: 3077-107.

[30] Eyre JR. A bias correction scheme for simulated TOVS brightness temperatures. ECMWF Tech Memo 1992; 186: 28 pp. [Available from European Centre for Medium-Range Weather Forecasts, Shinfield Park, Reading, Berkshire R62 9AX, United Kingdom.].

[31] Corbosiero KL, Wang W, Chen Y, Dudhia J, Davis C. Advance research WRF high resolution simulations of inner core structure of hurricanes KATRINA and RITA (2005). $8^{\text {th }}$ WRF users' workshop, National Center for Atmospheric Research, Boulder, CO. June 1115, 2007.

[32] Mathur MB. The National Meteorological Center's quasiLagrangian model for hurricane prediction. Mon Wea Rev 1991; 109: 1419-47.

[33] Kurihara Y, Ross RJ. An initialization scheme of hurricane models by vortex specification. Mon Wea Rev 1993; 121: 2030-45.

(C) Xu et al.; Licensee Bentham Open.

This is an open access article licensed under the terms of the Creative Commons Attribution Non-Commercial License (http://creativecommons.org/licenses/by$\mathrm{nc} / 3.0 /$ ) which permits unrestricted, non-commercial use, distribution and reproduction in any medium, provided the work is properly cited. 